\title{
Real-time X-ray diffraction measurements of structural dynamics and polymorphism in diindenoperylene growth
}

\author{
Stefan Kowarik • Alexander Gerlach • Stefan Sellner • \\ Leide Cavalcanti • Oleg Konovalov • Frank Schreiber
}

Received: 13 August 2008 / Accepted: 24 November 2008 / Published online: 23 December 2008

(C) The Author(s) 2008. This article is published with open access at Springerlink.com

\begin{abstract}
We investigate the temperature-dependent polymorphs in diindenoperylene (DIP) thin films on sapphire and silicon oxide substrates using in situ X-ray scattering. On both substrates the DIP unit cell is very similar to the high-temperature phase of bulk crystals, with the substrate stabilising this structure well below the temperature where a phase transition to a low-temperature phase is observed in the bulk. Lowering the substrate temperature for DIP growth leads to a change in molecular orientation and an additional polymorph appears, with both these effects being more pronounced on sapphire as compared to silicon oxide. Using real-time reciprocal-space mapping we observe an expansion of the in-plane unit cell during DIP growth, which may be due to changes in molecular orientation as well as strain in the first monolayers.
\end{abstract}

PACS 61.66.Hq $\cdot 61.05 . \mathrm{cp} \cdot 68.55 . \mathrm{am}$

Electronic supplementary material The online version of this article (http://dx.doi.org/10.1007/s00339-008-5012-2) contains supplementary material, which is available to authorized users.

S. Kowarik $(\bowtie) \cdot$ A. Gerlach · S. Sellner · F. Schreiber Institut für Angewandte Physik, Auf der Morgenstelle 10, 72076

Tübingen, Germany

e-mail: stefan.kowarik@berkeley.edu

Fax: +1-510-6434983

Present address:

S. Kowarik

Lawrence Berkeley National Laboratory, 1 Cyclotron Road,

Berkeley, CA 94720, USA

L. Cavalcanti $\cdot$ O. Konovalov

ESRF, 6 Rue Jules Horowitz, Boîte Postale 220, 38043 Grenoble

Cedex 9, France

\section{Introduction}

Organic semiconductor devices show great promise for electronic and optoelectronic applications and their function and efficiency improve continuously, as can be seen from other articles in this special issue. At the same time, many fundamental physical and chemical properties of conjugated materials are still not fully understood. In particular, a thorough understanding of organic thin-film growth poses challenges to theory and experiment because new degrees of freedom such as molecular orientation are not included in established growth theories [1-4].

In this work we study the semiconductor diindenoperylene (DIP, $\mathrm{C}_{32} \mathrm{H}_{16}$ ), which shows promising charge-carrier mobilities of up to $0.1 \mathrm{~cm}^{2} / \mathrm{V} \mathrm{s}[5,6]$. Its temperaturedependent bulk crystal structures have recently been solved [4] and the exciton diffusion length has been determined [7]. Optical properties of DIP thin films have been investigated with ellipsometry and theoretically explained $[8,9]$. Thin DIP films have been found to exhibit high structural order [10] but also rapid roughening after initial layer-by-layer growth [11-13]. Encapsulation of DIP thin films has been employed for increased device stability [14, 15], and contact formation as well as electronic structure at contacts [16-20] have been studied. Its molecular orientation on insulators $[12,21]$ and metals $[20,22,23]$ has been measured, and high-resolution transmission electron microscopy (TEM) [24] and scanning tunneling microscopy (STM) [25] images give insight into the molecular arrangement. Despite the progress made in characterisation of DIP thin films, there are still open questions regarding the influence of substrate temperature and substrate material on growth dynamics and film structure. Further, the thin-film unit cell on the commonly used gate insulators silicon oxide and sapphire is still unknown. 
In this paper we therefore apply real-time X-ray diffraction to determine the DIP thin-film unit cell, which we find to be very similar to the unit cell of the high-temperature bulk phase. Using fast real-time reciprocal-space mapping we follow the expansion of the in-plane unit-cell dimensions during growth. We find that the substrate stabilises the hightemperature bulk phase, even when lowering the substrate temperature to $223 \mathrm{~K}$, but the lower temperature leads to phase co-existence with two other structures in the film.

\section{Experimental}

DIP was purchased from Institut für PAH-Forschung (Greifenberg, Germany) and purified by gradient sublimation before use. Sapphire substrates ( $A$ plane, average terrace width well below $1000 \AA$ ) and $\mathrm{Si}(100)$ wafers covered by their native oxides were cleaned ultrasonically with acetone, isopropanol and ultra-pure water followed by heating to $700 \mathrm{~K}$ in vacuum. This cleaning procedure leads to reproducible substrate surfaces, with X-ray photoelectron spectroscopy (XPS) measurements showing that the main surface contamination is carbon at a coverage below 0.1 monolayers (ML). Thin DIP films were prepared by organic molecular beam deposition (OMBD) at a growth rate of $\sim 3 \AA / m i n$ under UHV conditions (base pressure $7 \times 10^{-10} \mathrm{mbar}$ ). The in situ X-ray measurements were carried out at beamline ID10B at the ESRF in Grenoble, France, with a wavelength of $0.964 \AA$, using a vacuum chamber dedicated to OMBD [26] equipped with a beryllium window, evaporation cells and a quartz-crystal microbalance. We found no structural damage of the organic thin films due to X-ray exposure over time scales of several hours, as verified by comparison of $\mathrm{X}$ ray reflectivity data on previously exposed and unexposed spots on the samples. To probe the lattice constants parallel to the sample surface, we used grazing incidence X-ray diffraction (GIXD) employing a scintillation counter $\left(q_{\|}\right.$denotes the in-plane momentum transfer). The GIXD measurements were performed at incident angles of $0.136^{\circ}$ for sapphire and $0.108^{\circ}$ for silicon. Since these angles are below the critical angles for the respective substrates, the X-rays only penetrate several tens of $\AA$ into the substrates so that the surface-sensitive GIXD measurement mainly probes the in-plane structure of the DIP film [27].

We also determined the out-of-plane structure by mapping the reciprocal space along both $q_{\perp}$ and $q_{\|}\left(q_{\perp}\right.$ denotes the out-of-plane momentum transfer) with a MarCCD area detector albeit at a lower resolution than the point-detector scan. Again grazing incidence angles were chosen for surface sensitivity, but in contrast to point detector scans with an integration range of $\Delta q_{\perp}=0.033 \AA^{-1}$ the camera also registers the crystal truncation rod diffraction along $q_{\perp}$ [28]. Importantly, the data acquisition with the area detector is possible with integration times of $60 \mathrm{~s}$ for a sub-monolayer (ML) down to $20 \mathrm{~s}$ for thicker films, which is much faster than GIXD measurements with a point detector.

\section{Results and discussion}

Figure 1 shows the GIXD intensity for DIP on sapphire as a function of $\left|q_{\|}\right|$measured by a detector scan as well as the corresponding map in both $q_{\perp}$ and $q_{\|}$obtained with the CCD detector. Data of DIP on silicon oxide (not shown) exhibit nearly identical positions of in-plane and out-of-plane Bragg reflections, so that we conclude that the unit-cell dimensions on both substrates are identical within our experimental accuracy (in the present setup $\Delta q_{\|}=0.01 \AA^{-1}$ ). From the higher resolution detector scan we calculate the in-plane unit-cell parameters $a, b$ and their respective angle $\gamma$ as shown in Table 1 and find them to be very similar to ones published before for the $\sigma$-structure in Ref. [20] for silicon oxide.

To solve the complete set of unit-cell parameters a large reciprocal-space section has to be measured as shown in Fig. 1a. For each in-plane reflection in Fig. 1b the corresponding crystal-truncation rod is visible in the $q_{\perp}$ direction, even including higher order reflections for the in-plane forbidden (01) peak.

Theoretical peak positions indicated in Fig. 1a by crosses were calculated using Mathematica [29] and fitted to the experimental peak positions. We find good agreement between simulation and experiment for the unit-cell parameters in Table 1; only slight deviations occur for higher order reflections due to the fact that the Ewald sphere cannot be considered planar across the full aperture of the area detector. Comparing the thin-film unit-cell dimensions for growth on sapphire at $403 \mathrm{~K}$ with the bulk crystal unit cells at $403 \mathrm{~K}$ and $423 \mathrm{~K}$ (see Table 1 and Ref. [4]), we find that the thin-film unit cell is very close to the high-temperature bulk phase containing two molecules, but very different from the bulk phase unit cell at the same temperature, which contains four DIP molecules [4]. Note that the reflections have been indexed according to the standard crystallographic nomenclature used in Ref. [4] for bulk crystals, which is at variance with previous thin-film indexing schemes in Refs. [12, 13, 20].

While in principle it is possible to obtain the unit-cell dimensions from the peak positions and the molecular orientation within the unit cell from the peak intensities [29], the data quality with the CCD acquisition is not good enough for a quantitative analysis of the molecular orientation, because the background in the CCD camera image is higher than the background in a detector scan. This is due to the lack of collimating slits for the CCD, which in the present setup leads to air scattering and diffraction rings of the $\mathrm{Be}$ 
Fig. 1 GIXD reflections for a 200-Å DIP film grown on sapphire at $403 \mathrm{~K}$. The bottom picture (b) shows a scan with a scintillation counter at fixed $q_{\perp}=0.031 \AA^{-1}$, while the top picture (a) shows the corresponding $q_{\perp}-q_{\|}$plane as seen with a CCD camera.

Marked with red crosses are the theoretical positions corresponding to the unit cell given in Table 1. Inset: molecular arrangement in the bulk high-temperature unit cell [4], which we find to be very similar to the thin-film unit cell
Table 1 Unit cell parameters of the thin-film phase and highand low-temperature bulk phases (after Reference [4])

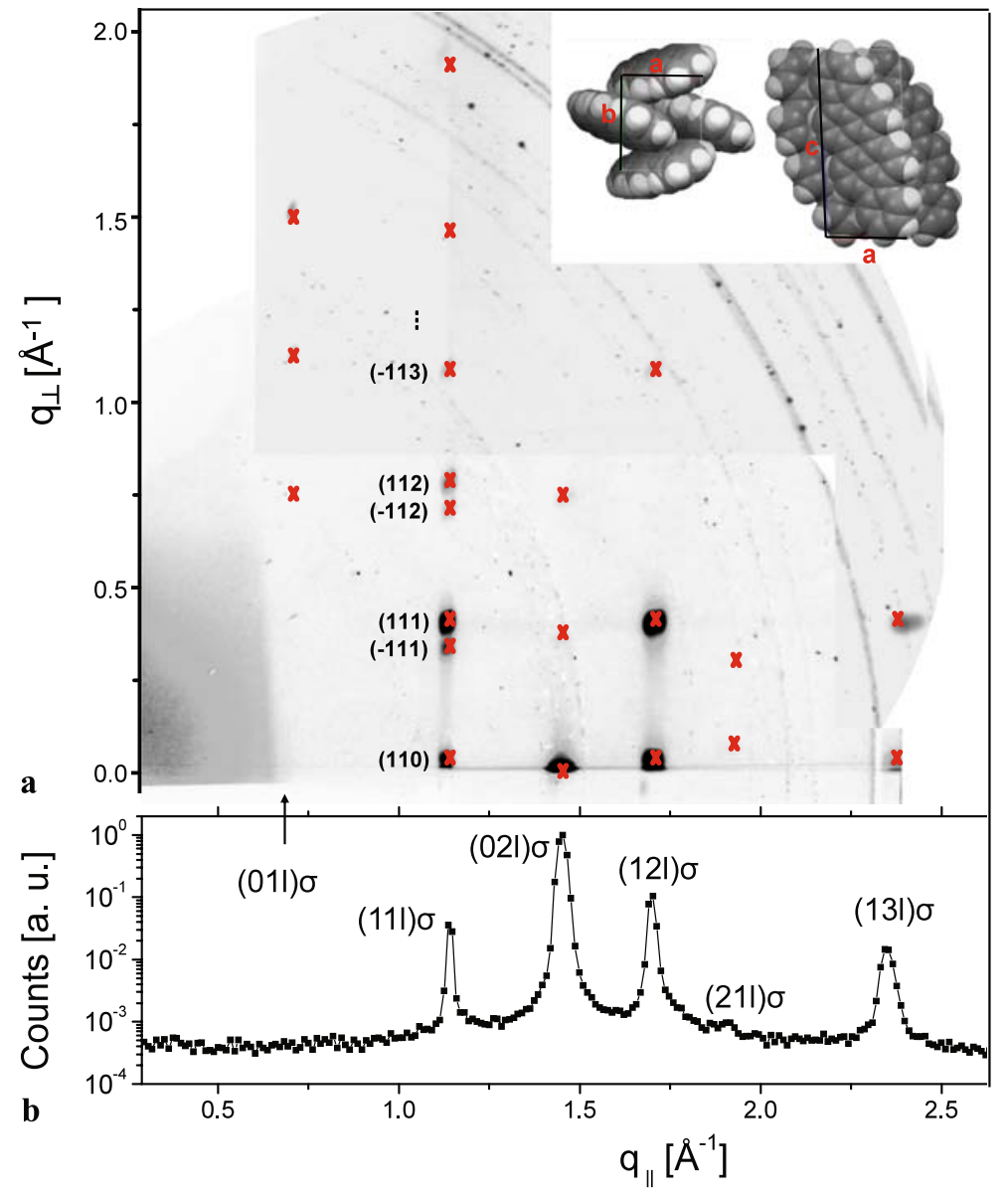

\begin{tabular}{|c|c|c|c|c|c|c|c|}
\hline$T[\mathrm{~K}]$ & $a[\AA]$ & $b[\AA]$ & $c[\AA]$ & $\alpha\left[^{\circ}\right]$ & $\beta\left[^{\circ}\right]$ & $\gamma\left[^{\circ}\right]$ & $V\left[\AA^{3}\right]$ \\
\hline \multicolumn{8}{|c|}{ Thin film $(\sigma)$} \\
\hline 403 & $7.09 \pm 0.04$ & $8.67 \pm 0.04$ & $16.9 \pm 0.5$ & 90.0 & $92.2 \pm 0.2$ & 90.0 & $1037 \pm 30$ \\
\hline \multicolumn{8}{|c|}{ Bulk crystal: } \\
\hline 423 & 7.1710 & 8.5497 & 16.798 & 90.0 & 92.416 & 90.0 & 1028.9 \\
\hline 403 & 11.6593 & 13.0103 & 14.966 & 98.441 & 98.024 & 114.549 & 1991.3 \\
\hline
\end{tabular}

window being visible in the CCD image but not in the detector scan. Even though we cannot determine the arrangement of the DIP molecules, the nearly identical unit-cell dimensions suggest, that the molecular packing is similar to the one in high-temperature bulk crystals, which is shown in the inset of Fig. 1a.

Apart from the unit-cell dimensions of the DIP crystallites, X-ray data also yield information on the arrangement of the crystallites on the surface. All the allowed in-plane reflections are found in a $2 \theta$ detector scan on both the singlecrystalline sapphire and amorphous silicon oxide substrates, so that we do not expect any preferential azimuthal orientation of DIP crystallites. While the in-plane order therefore is random (a $2 \mathrm{~d}$ powder), in out-of-plane X-ray reflectivity measurements we only find (001) reflections associated with a spacing of $d_{(00 l)}=16.6 \AA$ indicating a fibre-like structure of the $\sigma$-phase on sapphire and silicon oxide, that is the DIP material is textured with the long molecular axis nearly parallel to the surface normal (molecules standing upright, $\sigma$-structure) and the crystallites are rotated randomly around that axis.

For a closer look at changes of the unit-cell dimensions during growth of DIP on sapphire at $403 \mathrm{~K}$ we mapped a section of the reciprocal space covering crystal-truncation rods [28] of DIP in real time. Real-time measurements have to overcome the inherently weak signal-to-noise ratio of the thin films, while at the same time acquisition times have to be fast compared to the time to grow one monolayer 
Fig. 2 (a) Three representative images of a series of GIXD reciprocal-space maps acquired during growth of DIP on sapphire at $403 \mathrm{~K}$ substrate temperature. Already for a sub-monolayer in-plane reflections are visible, and with increasing coverage higher order reflections $(l=1,2)$ appear due to the multilayer out-of-plane periodicity (see supporting online material for full real-time video). (b) The in-plane reflections can be seen to shift during growth, which corresponds to an increase of the in-plane lattice parameters as shown in (c). Interestingly, this expansion is a collective change also of the first monolayer, as can be seen from the shift of the Bragg reflections as a whole. (d) Scattering geometry for real-time GIXD measurements; the incident angle $\alpha_{i}$ for the real-time measurement was $0.136^{\circ}$

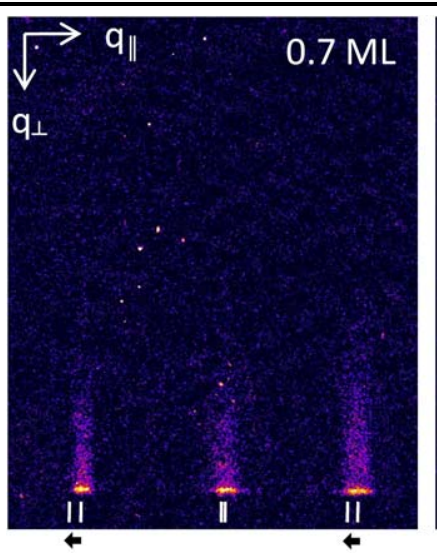

(11 I)

(02 I)

(12I)

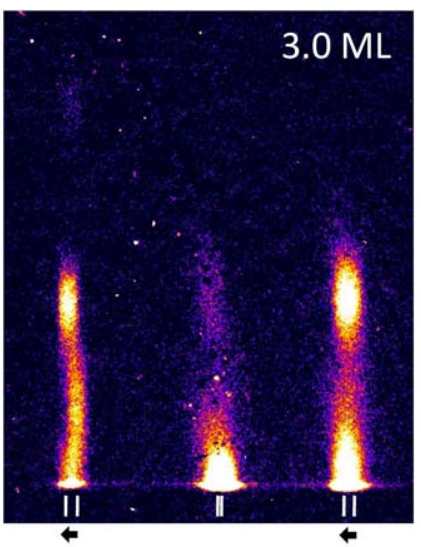

(11 I)

(02 I)

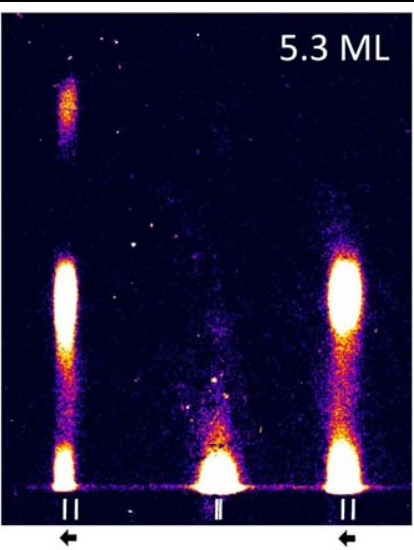

(11 I)

(02 I)

(12 I)

a

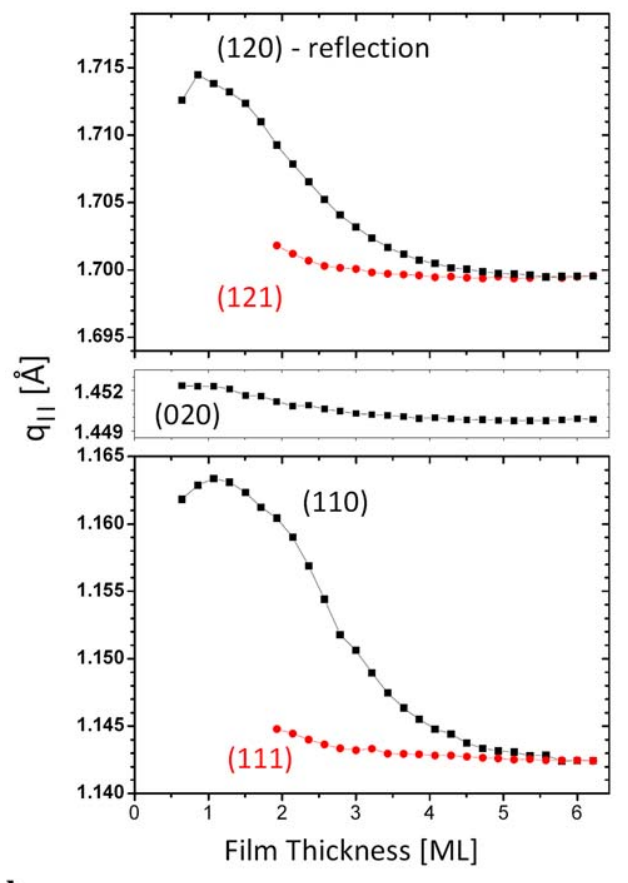

b
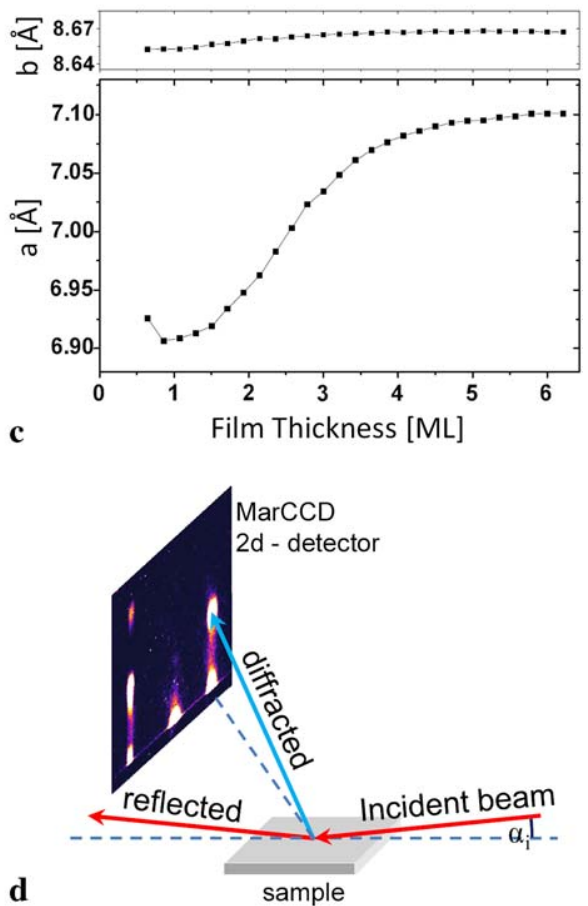

( $\sim 5$ min at a growth rate of $\sim 3 \AA / m i n)$. High X-ray flux at the ESRF, a static scattering geometry without mechanical scanning movements and parallel acquisition of a wide $q$ range with an area detector enable us to acquire realtime data with sub-ML sensitivity as shown in Fig. 2a (see supporting online material for a video of the full real-time growth sequence). Already for a sub-monolayer film the inplane reflections are clearly visible as DIP crystallites with a well-defined in-plane lattice and $1 \mathrm{ML}$ height form. Since the first ML does not exhibit any periodicity along the surface normal, no out-of-plane Bragg reflections are visible (only 'streaks' along $q_{\perp}$ ). After deposition of further MLs the out-of-plane periodicity develops and correspondingly Bragg reflections along the crystal-truncation rod form and become stronger. We note that the CCD images in Fig. 2a have lower background as compared to Fig. 1a, because the first frame of the time series at zero DIP sample coverage allows us to subtract the influence of air scattering and the diffraction from the beryllium window.

Interestingly, the in-plane reflections of DIP shift during growth revealing an expansion in unit-cell dimensions due to molecular re-arrangement such as a changing molecular tilt or relaxation of strain in the first ML. Note that the reflections move as a whole with increasing thickness, indicating that the structure changes in a relatively collective fashion, i.e. not only in the newly growing layer, but the structural change/strain relaxation also occurs in the layers underneath. Figure $2 b$ shows a quantitative analysis of the shift of the reflections visible in Fig. 2a for growth of the first six MLs. The (120) and (110) in-plane reflections show a pronounced shift while the (121) and (111) reflections, which stem from crystallites with a thickness of several MLs, only 


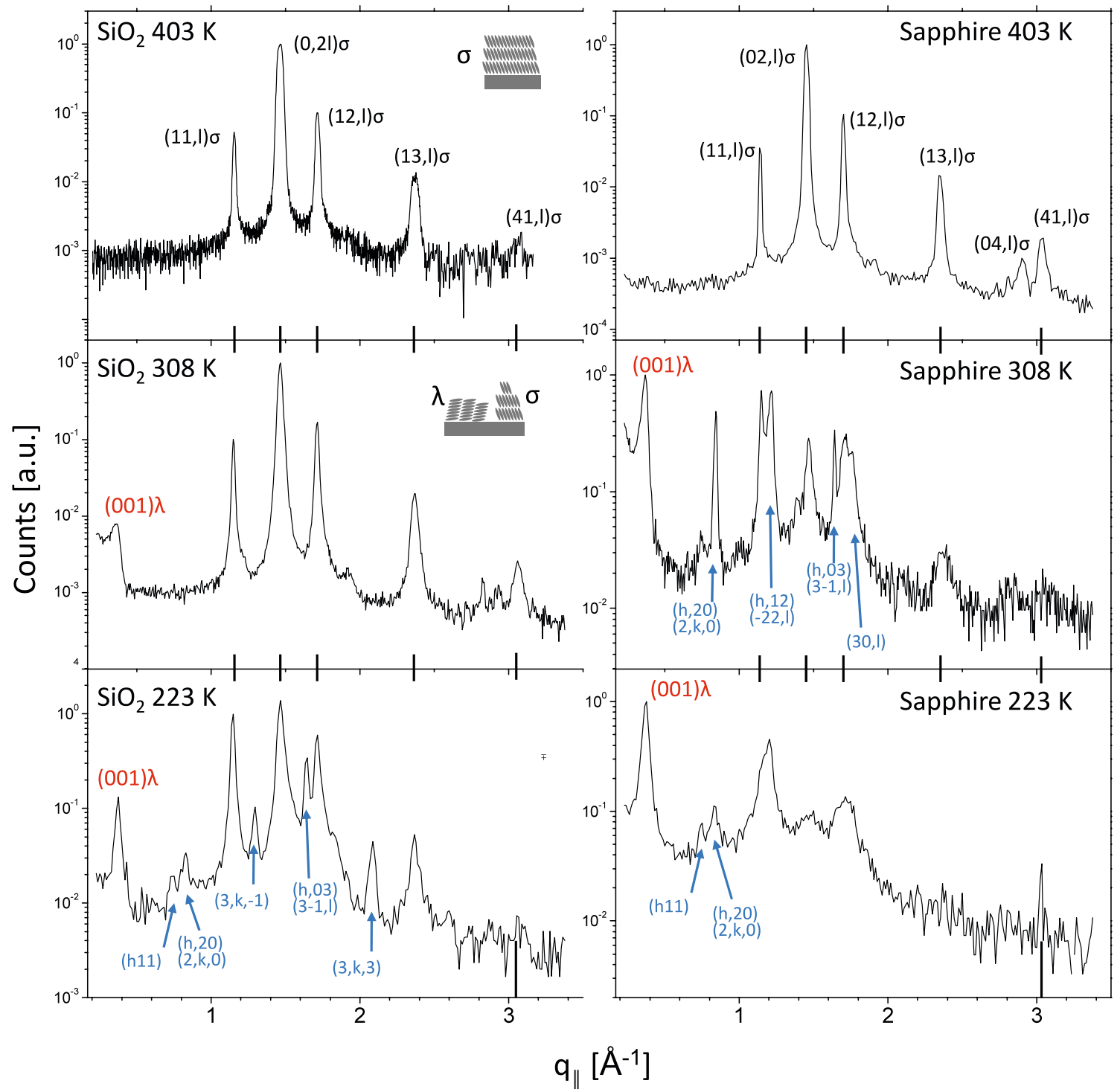

Fig. 3 In-plane reflection patterns for six DIP films of $\sim 200 \AA$ grown on silicon oxide and sapphire at different substrate temperatures. At high substrate temperature only the $\sigma$-structure grows, that is molecules are standing upright (for unit cell see Table 1). For lower substrate temperatures a structure with lying-down molecules ( $\lambda$-structure) also

exhibit a minor change. This demonstrates that thicker films quickly adopt a structure close to the high-temperature bulk phase while the structural changes occur mostly in the first ML. From the Bragg reflections the changes of the in-plane lattice constants $a$ and $b$ can be calculated as shown in Fig. 2c. While the $b$ axis only changes slightly, the $a$ axis expands by $2.6 \%$ during growth, which may be due to a compressively strained first monolayer, but can also be explained by a changing molecular tilt angle. Molecules in a more upright standing and therefore more densely packed first ML have been found to minimise the surface energy in pentacene films [30], and similarly one may hypothesise that develops and reflections of a third phase appear, which have been tentatively assigned to the low-temperature bulk phase. Both these structures are more pronounced on sapphire, where growth at $223 \mathrm{~K}$ predominantly occurs in the $\lambda$-structure and other Bragg reflections are broadened due to increased disorder

DIP molecules adopt a more upright orientation in the first ML.

Comparing the real-time GIXD measurement in Fig. 2 to previous work $[12,13]$ on structural changes during DIP growth, we note that the use of an area detector gives superior time resolution while also simultaneously yielding information about the in-plane and out-of-plane structures. The overall change is similar to the one observed in Ref. [13], with some differences due to the different resolutions of the two experimental setups and the continuous growth in this work as opposed to an interrupted growth mode, which can lead to different growth kinetics and de-wetting. 
Beyond growth at $403 \mathrm{~K}$ we investigated the dependency of the DIP thin-film structure on substrate temperature by recording GIXD reflections in situ on both silicon oxide and sapphire substrates. As shown in Fig. 3, growth on silicon oxide at $403 \mathrm{~K}$ reproduces the pure $\sigma$-phase growth as found for sapphire in Fig. 1 with a nearly identical diffraction pattern. Lowering the substrate temperature for growth to $308 \mathrm{~K}$, that is $100 \mathrm{~K}$ below the temperature for which a phase transition is observed in the bulk crystal [4], we still find that the film grows preferentially in the $\sigma$-structure. This demonstrates that the substrate stabilises the thin-film $\sigma$-structure far beyond the point where this unit cell in bulk undergoes a phase transition. An additional reflection at $q_{\|}=0.362 \AA^{-1}$ appears on silicon oxide at $308 \mathrm{~K}$, which is characteristic of the $\lambda$-structure in which molecules are 'lying down'. As reported in Ref. [20], the $\lambda$-structure is similar to the $\sigma$ structure, yet with the long molecular axis rotated into the surface plane. The $\lambda$-structure becomes more pronounced at $223 \mathrm{~K}$, but still the film predominantly grows in a 'standing upright' $\sigma$-structure. Interestingly, new reflections belonging to a third polymorph of DIP appear at $223 \mathrm{~K}$ on silicon oxide. It is plausible to assume that this new structure is similar to the low-temperature bulk structure and indeed it is possible to index the new reflections using this low-temperature bulk structure (see Table 1). It is necessary though to assume various orientations of the bulk polymorph (that is $a-c$ and $b-c$ planes parallel to the substrate surface) and due to the limited number of available reflections it is not possible to unambiguously assign them to the low-temperature bulk phase.

Growth on sapphire shows a similar trend when lowering the substrate temperature: the fraction of the $\sigma$-structure decreases while the $\lambda$-structure and the third polymorph become more pronounced. In comparison with silicon substrates, the latter two structures are more favoured and already at $308 \mathrm{~K}$ the $\lambda$-structure Bragg reflection is stronger than the $\sigma$-structure reflection and a third phase (again tentatively assigned to the low-temperature bulk phase) is already formed, in contrast to silicon oxide.

At $223 \mathrm{~K}$ the (001) reflection of the $\lambda$-phase is stronger than other reflections, demonstrating that the film predominantly grows in the $\lambda$-structure. All other reflections are weakened and broadened due to strong disorder in the film. Nevertheless, a reciprocal-space map for growth on sapphire at $223 \mathrm{~K}$ (not shown) reveals crystal-truncation rods for the $\sigma$-structure, demonstrating out-of-plane ordering even at low temperatures, and also new reflections at $q_{\perp}=0.94 \AA^{-1}$ and $q_{\|}=0.19 \AA^{-1}$ are visible that can be assigned to the (002) low-temperature bulk reflection, further supporting the assignment of the new reflections to the lowtemperature bulk phase. The fact that the lying-down structure is more pronounced on sapphire can be explained by the slightly larger van-der-Waals interaction and the higher step density leading to increased nucleation of crystallites with molecules in the $\lambda$-configuration, thereby maximising the substrate-molecule interaction. Further, decreased surface diffusion due to the step edges and stronger substrate interaction explain why growth on sapphire is similar to growth on silicon at lower substrate temperatures, that is freezing out diffusion processes on silicon and stronger interactions on sapphire have a similar effect.

\section{Conclusions}

We have investigated the temperature-dependent polymorphs in DIP thin films on sapphire and silicon oxide substrates. We find the unit cell of DIP thin films to be very similar to the high-temperature phase of bulk crystals, but the influence of the substrate stabilises this structure even $200 \mathrm{~K}$ below the temperature where a phase transition to a lowtemperature phase is observed in the bulk. While at high temperatures $(403 \mathrm{~K})$ only a single $\sigma$-structure of standing upright molecules is found, lower substrate temperatures $(<308 \mathrm{~K})$ lead to the occurrence of a $\lambda$-structure (lying-down molecules) as well as a third phase whose Bragg reflections can be explained by assuming that the lowtemperature bulk phase appears. We have used real-time and in situ reciprocal-space mapping to reveal an expansion of the in-plane unit-cell dimensions during growth of the first five DIP layers, which may be due to both changing molecular orientation and relaxation of a compressively strained first ML. The thickness range of these structural changes coincides with the thickness regime in which nearly perfect layer-by-layer growth occurs [12,13], indicating a connection between the breakdown of $2 \mathrm{~d}$ growth/onset of $3 \mathrm{~d}$ growth with the occurrence of a bulk-like thin-film structure. Further work and in particular theoretical studies of the bulk and surface energies in DIP thin films are needed for a comprehensive understanding.

Acknowledgements We want to thank J. Pflaum, B. Nickel and S. Schiefer for valuable discussions as well as the EPSRC and DFG for financial support.

Open Access This article is distributed under the terms of the Creative Commons Attribution Noncommercial License which permits any noncommercial use, distribution, and reproduction in any medium, provided the original author(s) and source are credited.

\section{References}

1. G. Witte, C. Wöll, J. Mater. Res. 19, 1889 (2004)

2. F. Schreiber, Phys. Status Solidi A 201, 1037 (2004)

3. S. Kowarik, A. Gerlach, F. Schreiber, J. Phys. Condens. Matter 20, 184005 (2008)

4. M.A. Heinrich, J. Pflaum, A.K. Tripathi, W. Frey, M.L. Steigerwald, T. Siegrist, J. Phys. Chem. C 111, 18878 (2007) 
5. N. Karl, K.H. Kraft, J. Marktanner, M. Munch, F. Schatz, R. Stehle, H.M. Uhde, J. Vac. Sci. Technol. A Vac. Surf. Films 17, 2318 (1999)

6. A.K. Tripathi, J. Pflaum, Appl. Phys. Lett. 89, 082103 (2006)

7. D. Kurrle, J. Pflaum, Appl. Phys. Lett. 92, 133306 (2008)

8. U. Heinemeyer, R. Scholz, L. Gisslen, M.I. Alonso, J.O. Osso, M. Garriga, A. Hinderhofer, M. Kytka, S. Kowarik, A. Gerlach, F. Schreiber, Phys. Rev. B 78, 085210 (2008)

9. L.M. Ramaniah, M. Boero, Phys. Rev. A 74, 042505 (2006)

10. A.C. Dürr, F. Schreiber, M. Munch, N. Karl, B. Krause, V. Kruppa, H. Dosch, Appl. Phys. Lett. 81, 2276 (2002)

11. A.C. Dürr, F. Schreiber, K.A. Ritley, V. Kruppa, J. Krug, H. Dosch, B. Struth, Phys. Rev. Lett. 90, 016104 (2003)

12. S. Kowarik, A. Gerlach, S. Sellner, F. Schreiber, L. Cavalcanti, O. Konovalov, Phys. Rev. Lett. 96, 125504 (2006)

13. X.N. Zhang, E. Barrena, D.G. de Oteyza, H. Dosch, Surf. Sci. 601, 2420 (2007)

14. S. Sellner, A. Gerlach, F. Schreiber, M. Kelsch, N. Kasper, H. Dosch, S. Meyer, J. Pflaum, M. Fischer, B. Gompf, Adv. Mater. 16, 1750 (2004)

15. S. Sellner, A. Gerlach, S. Kowarik, F. Schreiber, H. Dosch, S. Meyer, J. Pflaum, G. Ulbricht, Thin Solid Films 516, 6377 (2008)

16. A.C. Dürr, F. Schreiber, M. Kelsch, H.D. Carstanjen, H. Dosch, O.H. Seeck, J. Appl. Phys. 93, 5201 (2003)

17. A.C. Dürr, F. Schreiber, M. Kelsch, H.D. Carstanjen, H. Dosch, Adv. Mater. 14, 961 (2002)

18. M. Scharnberg, R. Adelung, F. Faupel, Phys. Status Solidi A Appl. Mater. Sci. 205, 578 (2008)
19. N. Koch, A.C. Dürr, J. Ghijsen, R.L. Johnson, J.J. Pireaux, J. Schwartz, F. Schreiber, H. Dosch, A. Kahn, Thin Solid Films 441, 145 (2003)

20. A.C. Dürr, N. Koch, M. Kelsch, A. Rühm, J. Ghijsen, R.L. Johnson, J.J. Pireaux, J. Schwartz, F. Schreiber, H. Dosch, A. Kahn, Phys. Rev. B 68, 115428 (2003)

21. A.C. Dürr, B. Nickel, V. Sharma, U. Taffner, H. Dosch, Thin Solid Films 503, 127 (2006)

22. M.B. Casu, I. Biswas, B.E. Schuster, M. Nagel, P. Nagel, S. Schuppler, T. Chasse, Appl. Phys. Lett. 93, 024103 (2008)

23. M.B. Casu, I. Biswas, M. Nagel, P. Nagel, S. Schuppler, T. Chasse, Phys. Rev. B 78, 075310 (2008)

24. A. Hoshino, S. Isoda, T. Kobayashi, J. Cryst. Growth 115, 826 (1991)

25. D.G. de Oteyza, E. Barrena, M. Ruiz-Oses, I. Silanes, B.P. Doyle, J.E. Ortega, A. Arnau, H. Dosch, Y. Wakayama, J. Phys. Chem. C 112, 7168 (2008)

26. K.A. Ritley, B. Krause, F. Schreiber, H. Dosch, Rev. Sci. Instrum. 72, 1453 (2001)

27. H. Dosch, Critical Phenomena at Surfaces and Interfaces: Evanescent X-ray and Neutron Scattering (Springer, Berlin, 1992)

28. J. Als-Nielsen, D. McMorrow, Elements of Modern X-Ray Physics (Wiley, New York, 2001)

29. S. Schiefer, M. Huth, A. Dobrinevski, B. Nickel, J. Am. Chem. Soc. 129, 10316 (2007)

30. L.F. Drummy, D.C. Martin, Adv. Mater. 17, 903 (2005) 